Dieser Aufgabe widmet sich Mechthild Frentrup in ihrer Dissertation am Department für Agrarökonomie der Georg-August-Universität Göttingen. Sie entwickelt ein Konzept zur Messung der Transparenz in Wertschöpfungsketten des Agribusiness, das die kommunikativen und strategischen Elemente von Transparenz in den Mittelpunkt rückt, und erprobt dieses am Beispiel der Supply Chains der deutschen Milch- und Fleischwirtschaft. Das entwickelte Modell ermöglicht es den Unternehmen des Agribusiness, den Status quo von Transparenz in der Wertschöpfungskette zu erfassen und die Situation im eigenen Unternehmen mit anderen Unternehmen zu vergleichen. Daraus ergeben sich für das praktische Management wichtige Ansatzpunkte, um aus der Verpflichtung zu mehr Transparenz einen Vorteil im Wettbewerb zu entwickeln und die ökonomischen Ziele des Unternehmens mit der zunehmend deutlich geforderten Gesellschaftsorientierung des Agribusiness zu vereinbaren.

Für Akteure innerhalb der Wertschöpfungskette des Lebensmittelhandels bietet die Dissertation zahlreiche Anregungen und Handlungshinweise, wie gemeinsam eine transparentere und nachhaltigere Supply Chain aufgebaut und kommuniziert werden kann.

N. Meise

\section{Medien im Marketing}

Gröppel-Klein, Andrea/Germelmann, Claas Christian (Hrsg.): Medien im Marketing - Optionen der Unternehmenskommunikation, Wiesbaden: Gabler, 2009, 553 Seiten, EUR (D) 69,90, ISBN 978-3-8349-0735-6.

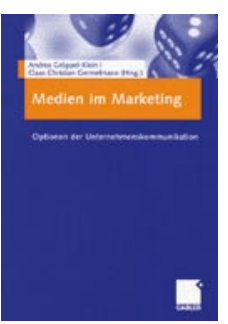

Die Unternehmen sehen sich heute bei der GestaltungihrerUnternehmenskommunikation einer Vielzahl von Medienoptionen gegenübergestellt. Die Selektion adäquater Kommunikationskanäle ist jedoch in Zeiten von Informationsüberflutung und mangelnder Wahrnehmung von höchster Bedeutung, um die Botschaft zur jeweiligen Zielgruppe zu transportieren. Gleichzeitig besitzen die Medien eine wachsende Bedeutung im Leben der Konsumenten, da sie immer noch wesentlich zur Meinungsbildung beitragen. Das Herausgeberwerk von GröppelKlein und Germelmann betont in besonderem Maße den für die Zukunft zunehmend notwendigen Dialog zwischen der Marketingund der Medienwissenschaft, um den Herausforderungen begegnen zu können, und zeigt Wege zur Navigation in dieser vielfältigen und unübersichtlichen Medienlandschaft auf.

Das Buch ist in sechs zentrale Kapitel untergliedert. In Teil 1 wird „Die Bedeutung der Medienperspektive für das Marketing“ aus unterschiedlichen Blickrichtungen erläutert. Dabei werden insbesondere das $\mathrm{Zu}$ sammenwirken und die Notwendigkeit zur integrierten Betrachtungsweise der beiden Disziplinen betont. Die Wirkung unterschiedlicher Medien und einzelner Instrumente auf das Konsumentenverhalten wird in den Beiträgen in Kapitel 2 untersucht. Der dritte Abschnitt ist der Unternehmenskommunikation gewidmet. Die Autoren setzen sich mit der Wirkung verschiedener Medien, beispielsweise im Bereich PR oder Krisenkommunikation, auseinander. Die Rahmenbedingungen der Medienwirkung werden im anschließenden Abschnitt thematisiert. Dabei stehen insbesondere Maßnahmen, die zur Entstehung von Engagement, zum Aufbau von Glaubwürdigkeit und zur Bildung von Vertrauen beitragen, im Mittelpunkt der Diskussion. Kapitel 5 umfasst die Konsequenzen für die Marketingorganisation und Marketingplanung, die durch die Inkorporation der Medienperspektive entstehen. Abschließend beschäftigt sich Teil 6 mit den Anwendungsfeldern für Medien im Marketing. Die Bedeutung der Medien im Einzelhandel wird dabei ebenso berücksichtigt, wie der Einfluss den Medien auf die Film- oder die Sportindustrie ausüben.

Gröppel-Klein und Germelmann gelingt dabei nicht nur eine praxisorientierte, sondern auch eine wissenschaftlich fundierte Betrachtung der Bedeutung, Möglichkeiten und Herausforderungen bei der Integration von Marketing und Medien. Der Sammelband eignet sich somit nicht nur für Führungskräfte im Bereich der Marketingkommunikation, sondern auch für Studierende mit dem Schwerpunkt Marketing und Medienmanagement sowie für Wissenschaftler, die sich mit den integrierten Fragestellungen von Marketing, Medien und PR auseinandersetzen möchten.

V.Walter

\section{Marketing}

Jacob, Frank: Marketing - Eine Einführung für das Masterstudium, Stuttgart: W. Kohlhammer, 2009, 224 Seiten, EUR (D) 34,90, ISBN 978-3-17-020705-9

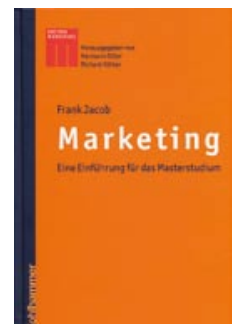

Im Marketing geht es im Wesentlichen um die Entwicklung und Förderung vorteilhafter Austauschbeziehungen zwischen Anbietern und Nachfragern. Die Marktorientierung, das heißt die Ausrichtung des gesamten Unternehmens am $\mathrm{Ab}$ satzmarkt im Allgemeinen und die Orientierung an den Kundenwünschen im Speziellen, stellt hierfür eine unverzichtbare Prämisse dar. Nur so kann das langfristige Überleben eines Unternehmens am Markt sichergestellt werden.

Jacob greift die Perspektive der Austauschorientierung im Marketing als Grundkonzept auf und stellt dementsprechend die Bedeutung und das Management von Transaktionen, Märkten und Beziehungen in den Mittelpunkt seines Lehrbuchs. In acht Kapiteln wird der Leser kompakt und umfassend in die Grundlagen des Marketing eingeführt. Der einleitende Abschnitt setzt sich mit den Marktstrukturen und Austauschprozessen sowie mit der gezielten Herbeiführung von Anbieter- und Kundenvorteilen auseinander. Das Verhalten der Marktakteure und insbesondere die Determinanten Unsicherheit und $\mathrm{Zu}$ friedenheit stehen im Zentrum der Ausführungen in Kapitel 2. Während sich Abschnitt 3 auf der individuellen Ebene mit der Identifikation relevanter Nutzenmerkmale und deren Messung befasst, werden mit der Heterogenität auf Märkten in Kapitel 4 Analyseverfahren zur Segmentierung der Nachfragergesamtheit betrachtet. In Abschnitt 5 widmet sich Jacob den Besonderheiten von Dienstleistungen als Austauschgegenstand und hebt die ökonomische Bedeutung von Services im Marketing hervor. Stand die Analyseaufgabe des Marketing im Fokus der vorliegenden Kapitel (insbesondere 2 bis 4), befassen sich die nun folgenden Kapitel 6, 7 und 8 mit den Handlungsebenen im Marketing. $\mathrm{Zu}$ erst werden die Bedeutung sowie die Grundlagen zum Management von Markttransaktionen dargestellt, die insbesondere durch die Informationsasymmetrie zwischen Anbie- 